\title{
Searching Form-Closure Fixturing Points on Objects Described by Triangular Meshes*
}

\author{
R. Suárez and J. Rosell \\ Institute of Industrial and Control Engineering (IOC) \\ Technical University of Catalonia (UPC), Barcelona, Spain \\ Emails: raul.suarez@upc.edu,jan.rosell@upc.edu
}

\begin{abstract}
The paper deals with the problem of finding a form-closure fixturing of objects modeled with triangular meshes and considering as quality measure the maximum wrench that the object can resist in any direction. Although a triangular mesh is a polyhedral representation of the object, the number of faces is too large to allow a practical application of existing approaches for polyhedral objects, and therefore some search procedure have to be applied. In the proposed approach the search of contact points is done looking for points directly on the object boundary instead of on the wrench space. In this way, all the object surface is homogeneously considered, while the quality is evaluated in the wrench space. The procedure iteratively looks, using heuristic criteria, for sets of points that improve the quality. The procedure was implemented and some application examples are included in the paper to illustrate the performance.
\end{abstract}

Index Terms-Grasp planning, fixturing, optimization, sampling.

\section{INTRODUCTION}

Fixing objects such that they can resist external perturbations without changing their positions is a quite relevant action in a number of tasks in robotics and automation. Typical examples where the object position must be kept when a perturbation force acts on it are grasping (the object position with respect to the grasp device must not change when, for instance, it collides with the environment), and fixturing in production lines (the object position with respect to the fixturing device must not change when, for instance, forces are applied on it by manufacturing tools or due to the assembly with another object).

The constraint of an object position can be done satisfying any of the following two properties: form-closure (the position of the fixtures/fingers ensures the object immobility) and force-closure (the forces applied by the fixtures/fingers ensure the object immobility) [1]. The force-closure constraint is more frequently required in grasping, since the movement of the object makes its own weight to act as an external perturbation, while the form-closure constraint is more frequently required in fixturing, where the object usually lies in a stable position while no operation in being performed on it.

${ }^{*}$ This work was partially supported by the CICYT projects DPI2004-03104 and DPI2005-00112, and Acción Integrada HI2005-0290.
The constraint of an object has been addressed considering different situations [2], mainly characterized by the dimension of the object (2D or 3D objects), type of object to be constrained (polyhedral or non-polyhedral), type of contact between the finger and the object (frictionless, frictional or soft contact), and the number of fingers (for 3D objects: equal or larger than 2 when soft contacts are considered, and equal or larger than 3 for frictional contacts, and equal or larger than 7 for frictionless contacts). Non-polyhedral objects are frequently modeled with a finite (but large) number of points, either using clouds of points as samples of the object surface or, for instance, triangular meshes approximating the object boundary (this representation is sometimes also used for polyhedral objects when the exact model is not available). There are a number of works covering all these cases, like, for instance: 2D polygonal objects [3], 2D nonpolygonal objects [4], 2D discrete objects [5], 3D polyhedral objects [6], [7], 3D non-polygonal objects [8], [9], and 3D discrete objects [10], [11].

This paper deals with the problem of finding form-closure constraint points on 3D objects described by triangular meshes. Even when a mesh can be considered a polyhedral representation of the object, the number of faces is too large to allow a practical application of existing approaches for polyhedral objects. Also, although some optimization criteria are applied in the search of the constraint points, it is not possible to assure the obtention of the optimal ones in a reasonable time, due to the exponential complexity of the problem and the large number of triangles. As it was already mentioned above, in order to obtain a form-closure constraint of a 3D object (i.e. without care about friction) seven is the minimum number of necessary frictionless contacts, and therefore seven frictionless contacts are considered in this work.

\section{PROPOSED APPROACH}

The idea behind the proposed approach is to find a solution searching the constraint points over all the object surface, instead of just search the corresponding wrench space which may lead to a solution with all the constraint points concentrated on a portion of the object.

The following nomenclature will be used to described the proposed approach: 
$\boldsymbol{p}_{i}$ : point on the object boundary

$\boldsymbol{n}_{i}$ : unitary inward normal to the object boundary at $\boldsymbol{p}_{i}$.

$\boldsymbol{f}_{i}$ : the contact force applied at $\boldsymbol{p}_{i}$.

$\boldsymbol{\tau}_{i}$ : torque produced by $\boldsymbol{f}_{i}$ (if the same reference system is used to describe $\boldsymbol{p}_{i}$ and to compute $\boldsymbol{\tau}_{i}$ then $\left.\boldsymbol{\tau}_{i}=\boldsymbol{p}_{i} \times \boldsymbol{f}_{i}\right)$

$\boldsymbol{w}_{i}=\left(\begin{array}{ll}\boldsymbol{f}_{i}^{T} & \lambda \boldsymbol{\tau}_{i}^{T}\end{array}\right)^{T}$ : wrench produced by $\boldsymbol{f}_{i}$ at $\boldsymbol{p}_{i}(\lambda$ is a constant to adjust the metric of the wrench space).

$P=\left\{\boldsymbol{p}_{i}, i=1, \ldots, n\right\}$ : set of $n$ known points on the object boundary

$W=\left\{\boldsymbol{w}_{i}, i=1, \ldots, n\right\}$ : set of the $n$ wrenches produced at the points in $P$.

$G p=\left\{\boldsymbol{p}_{1}, \ldots, \boldsymbol{p}_{7}\right\}$ : set of 7 points from $P$.

$G w=\left\{\boldsymbol{w}_{1}, \ldots, \boldsymbol{w}_{7}\right\}$ : set of 7 wrenches from $W$ corresponding to the points in $G p$.

With this nomenclature the problem can be formally stated as the search of a set $G p$ that allows a form-closure fixturing of the object with a good quality, which is also equivalent to the search of the corresponding set $G w$. The way the quality of the fixturing is evaluated is described below in Section II-C.

The search of the contact points is done with the following basic algorithm:

Step 1: Generate an initial set of seven points $G p$ and evaluate its quality.

Step 2: Select another point $\boldsymbol{p}_{j}$ on the object surface.

Step 3: Select a particular point $\boldsymbol{p}_{i} \in G p$

Step 4: Evaluate the resultant quality when $\boldsymbol{p}_{i}$ is replaced by $\boldsymbol{p}_{j}$ in $G p$.

Step 5: If the quality grows then update $G$ replacing $\boldsymbol{p}_{i}$ by $\boldsymbol{p}_{j}$.

Step 6: While a finishing condition is not satisfied goto step 2.

The finishing condition in step 6 of the algorithm can be:

- A given desired minimum quality is obtained.

- A given number of steps without improving the quality were performed.

- A given number of points on the object surface were visited.

- All the points on the object surface were visited.

The algorithm itself is a classical one, being the originality of the work the way each step is performed, which is described in the following subsections. As a difference with other approaches (e.g. [10]), here the algorithm continues the search for a grasp quality improvement once the first valid grasp was found.

\section{A. Selection of points on the object surface (Steps 1 and 2)}

The same selection procedure is used to generate the initial set of points in $G p$ (step 1) and the rest of the points
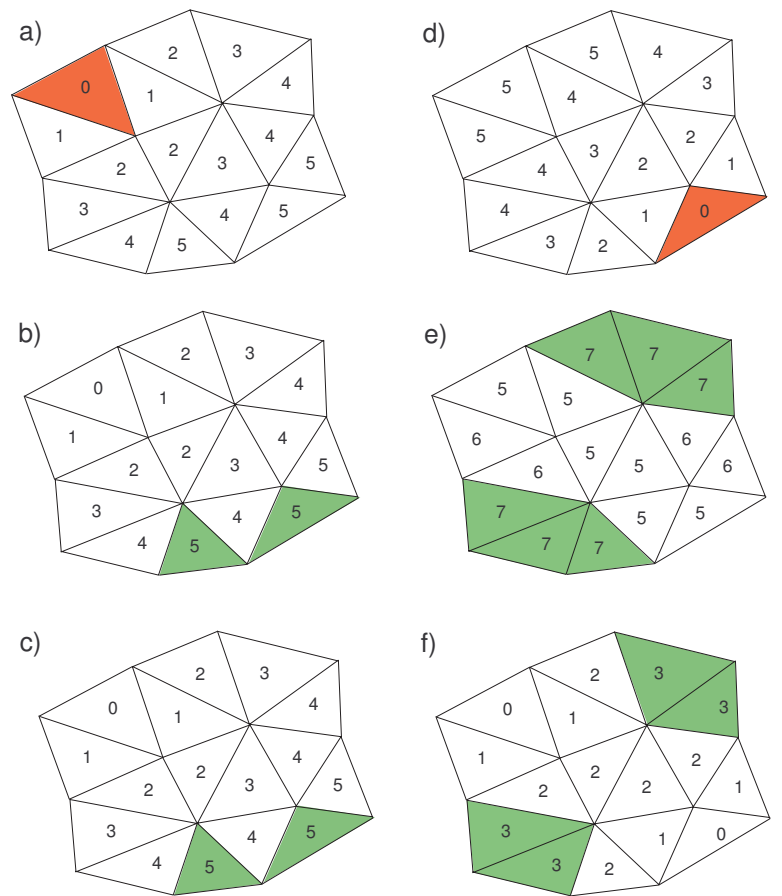

Fig. 1. The number in each triangle indicates: (a) and (d) Distance to the selected ones (colored); (b) Potential $V_{i}$ when the triangle of Figure a is the first to be selected; (c) Corresponding potential $U_{i}$; (d) Potential $V_{i}$ when the two triangles of Figure a and d are the ones selected; (f) Corresponding potential $U_{i}$.

during the search (step 2). The basic idea is to select points trying to uniformly cover the object surface.

The covering of the object surface is carried out using a deterministic sampling sequence that places each point of the sequence as far as possible from all the previous sampled points. Since each point is placed at the barycenter of a triangle in the mesh used to describe the object, a sequence of points can be considered as a sequence of triangles. The following assumptions about these triangular meshes are considered:

- The triangles in the mesh have similar size; when this is not the case the triangular meshes are preprocessed to obtain triangles with a similar area.

- The number of triangles in the mesh is large enough to properly describe the object.

- The distance between two triangles in the mesh is measured as the minimum number of triangle edges that must be crossed in a continuous path that connects them (passing through triangle vertices is disallowed).

The sampling procedure selects the triangle of the mesh that is the farthest from all the previous triangles of the sequence. Let:

- $\Delta V_{i}^{c}$ be the distance from triangle $i$ to the last sampled triangle. It is computed by propagation as shown in Figures 1a and 1d.

- $V_{i}$ be the summation of the distances from triangle $i$ to all the already sampled triangles (Figure 1b shows 

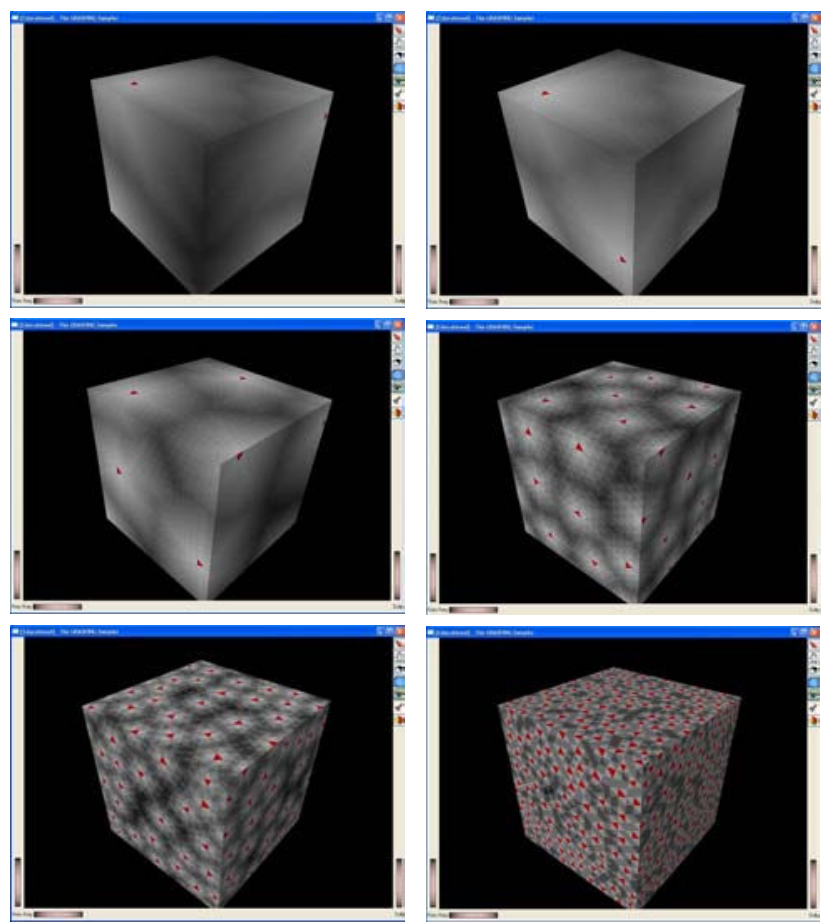

Fig. 2. Deterministic sampling of the surface of a cube (using 4, 5, 10, 50,150 and 500 samples from left-top to bottom-right).

$V_{i}$ for the single triangle of Figure 1a; Figure 1e shows $V_{i}$ when the triangles of Figures 1a and 1d have been selected).

- $U_{i}$ be the minimum distance from the triangle $i$ to the set of the already sampled triangles (Figures $1 \mathrm{c}$ and $1 \mathrm{f}$ correspond, respectively, to the situations of $1 \mathrm{~b}$ and $1 \mathrm{e})$.

Then, the sampling procedure to iteratively select the triangles in the mesh (and therefore the corresponding wrenches) is as follows:

1) Randomly choose the first triangle of the sequence and mark it as the current triangle $c$.

2) For each remaining triangle $i$ of the mesh:

a) Compute the distance $\Delta V_{i}^{c}$ to triangle $c$.

b) Update $V_{i}: V_{i}=V_{i}+\Delta V_{i}^{c}$.

c) If $U_{i}>\Delta V_{i}$ then $U_{i}=\Delta V_{i}^{c}$.

3) Choose the triangles with the highest $V_{i}$ (colored triangles in Figure $1 \mathrm{~b}$ and $1 \mathrm{e}$ ).

4) Among them choose the one with the highest $U_{i}$. If several triangles have the same value of $U_{i}$ (as the colored triangles in Figure 1c and 1f) randomly select one of them.

5) Add the chosen triangle to the sequence and mark it as the current triangle.

6) If there are still non-visited triangles in the mesh goto 2 .

7) Return.

The six snapshots of Figure 2 show the covering of the surface of a cube as the sampling sequence is executed. It illustrates how the surface is incrementally and uniformly
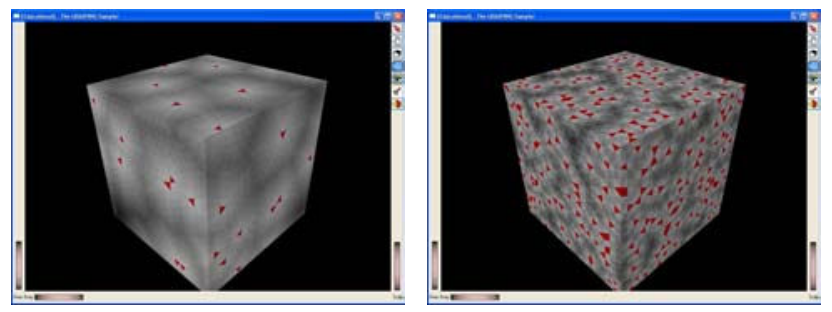

Fig. 3. Random sampling of the surface of a cube: 150 (left) and 500 samples (right).
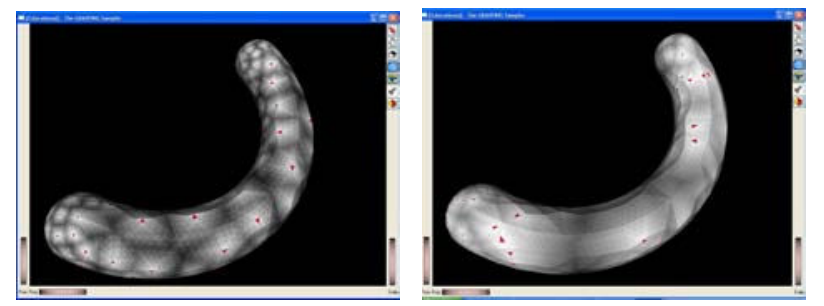

Fig. 4. Deterministic sampling (left) and random sampling (right) of the surface of a sausage using 150 samples.

covered. The already selected triangles are red while for the remaining triangles the higher $V_{i}$ the darker the grey. Just to compare the sequence obtained with this procedure with a pure random selection of triangles in the mesh with uniform distribution, Figure 3 shows two snapshots of the random sampling of the surface of the cube for 150 and 500 samples, and Figure 4 shows the comparison between the deterministic and the random sampling of the surface of a sausage for the first 150 samples.

\section{B. Selection of the point of Gp to be replaced (Step 3)}

Given a new point $\boldsymbol{p}_{j}$ (obtained from Step 2) the selection of the point $\boldsymbol{p}_{i} \in G p$ to be replaced is done using a criterion that avoids checking for a local optimal replacement among the seven points in $G$ and reduces the computational cost of the selection. The relevant property of the seven points $\boldsymbol{p}_{i} \in G p$ that makes them to define a form-closure grasp (FC-grasp for short) is the relative directions of the wrenches $\boldsymbol{w}_{i} \in G w$. The module of $\boldsymbol{w}_{i}$ may affect the quality of the fixturing produced by $G w$ but is irrelevant for the existence or not of a FC-grasp. Considering this property, the point $\boldsymbol{p}_{i} \in G$ whose associate wrench $\boldsymbol{w}_{i}$ has the closest direction to the direction of the wrench $\boldsymbol{w}_{j}$ associated with the new point $\boldsymbol{p}_{j}$ is selected as candidate point to be replaced in $G p$, i.e. the candidate point is the point $\boldsymbol{p}_{i}, i=1, \ldots, 7$, that minimizes

$$
\arccos \left(\frac{\boldsymbol{w}_{i} \cdot \boldsymbol{w}_{j}}{\left\|\boldsymbol{w}_{i}\right\|\left\|\boldsymbol{w}_{j}\right\|}\right)
$$

This criterion minimizes the change in the directions of the wrenches in $G w$.

\section{Evaluation of the quality (Step 4)}

The evaluation of the quality of a set $G p$ in Step 4 is done in the following way. When the set $G p$ produces a 
FC-grasp, the quality is equivalent to the largest perturbation wrench that the grasp can resist with independence of its direction [12], [13]. Calling $\mathcal{P}$ to the set of wrenches that the forces applied by the fingers can generate, the quality can be expressed as

$$
Q=\min _{\boldsymbol{\omega} \in \partial \mathcal{P}}\|\boldsymbol{\omega}\|
$$

with $\partial \mathcal{P}$ being the boundary of $\mathcal{P}$. The geometrical interpretation of the grasp quality is that it is equivalent to the radius of the largest 6-dimensional ball centered at the origin of the wrench space and fully contained in $\mathcal{P}$, thus, the criterion is also known as the criterion of the maximum ball. This is one of the most popular quality measures (examples of its use can be found in [14-16]) because it is independent of any external perturbation. In the case that the directions of the expected perturbations were known a variation of the quality measure optimizing the resistance along these directions can be considered, like for instance the Q-distance presented in [8].

In order to speed up the search of a good grasp, here we also considered a quality measure for the sets $G p$ that does not produce a FC-grasp; in these cases, the quality defined above should be zero, since the origin is outside $\mathcal{P}$, nevertheless we consider a negative quality defined by

$$
Q=-\min _{\boldsymbol{\omega} \in \partial \mathcal{P}}\|\boldsymbol{\omega}\|
$$

The geometrical interpretation in this case is that the quality is equivalent to the radius of the largest 6-dimensional ball centered at the origin of the wrench space and fully outside $\mathcal{P}$, indicating in some way, how far is the grasp from being a FC-grasp.

The shape of $\mathcal{P}$ depends on the constraints imposed on the finger forces [17]. Here one of the simplest and frequently considered constraints is used. It is assumed that the sum of the modules of the forces applied by the 7 fingers is limited (for instance due to a limited common power source for all the fingers actuators), i.e. $\sum_{i=1}^{n}\left\|\boldsymbol{f}_{i}\right\| \leq f_{\max }$ (without loss of generality and just to simplify the implementation, it is considered that $f_{\max }=1$ ). Under this assumption, $\mathcal{P}$ is the 6-dimensional polytope defined in the wrench space by the convex hull of the seven applied wrenches, i.e.

$$
\mathcal{P}=\operatorname{ConvexHull}\left(\boldsymbol{w}_{1}, \ldots, \boldsymbol{w}_{7}\right)
$$

Then, each face of the polytope is defined by 6 of the wrenches applied by the fingers, and the quality $Q$ of the set $G p$ is equivalent to the distance from the origin of the wrench space to the boundary of $\mathcal{P}$, with positive sign if $G p$ produces a FC-grasp and negative sign otherwise. Figure 5 gives qualitative examples in a hypothetical 2-dimensional wrench space of the polytope $\mathcal{P}$ (in this case a polygon) and the quality $Q$ as the radius of the maximum balls (in this case a circumference) for a FC-grasp (positive $Q$ ) and a non FC-grasp (negative $Q$ ) .

In this situation, the procedure used to evaluate the quality $Q$ produced by a set $G w$ is the following:
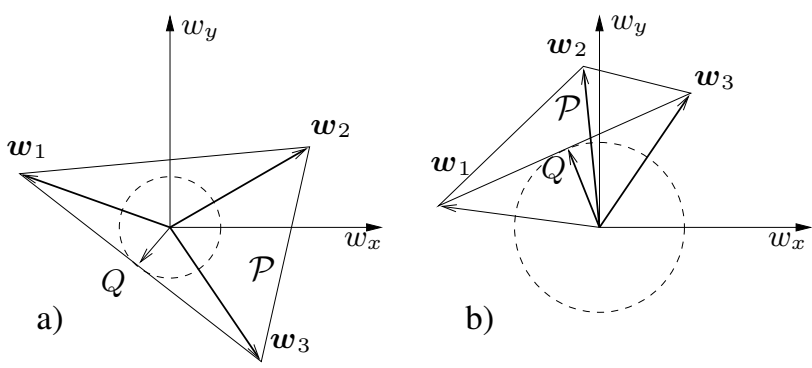

Fig. 5. Qualitative bidimensional example of $\mathcal{P}$ and the grasp quality $Q$ using three fingers: a) a FC-grasp $(O \in \mathcal{P}$ then $Q>0)$; b) a non FC-grasp $(O \notin \mathcal{P}$ then $Q<0)$.

1) For $i=1, \ldots, 7$ generate the hyperplane $\mathcal{H}_{i}$ defined by the subset $G w \prime_{i} \in G w$ of 6 wrenches that does not contain the element $w_{i}$.

a) Compute the distance $d_{i}$ from the origin $O$ to $\mathcal{H}_{i}$.

b) If $w_{i}$ and $O$ lie in the same half-space defined by $\mathcal{H}_{i}$ then $Q_{a u x_{i}}=d_{i}$ else $Q_{a u x_{i}}=-d_{i}$.

2) If $\forall i, Q_{\text {aux }_{i}}>0$ then $Q=\min \left\{Q_{a_{u x}}\right\}$, for $\mathrm{i}=1, \ldots, 7$ else $Q$ is the distance from $O$ to ConvexHull $(G w)$.

\section{EXAMPLES}

The proposed approach was implemented and tested on different objects producing satisfactory results. In all the examples the best results were obtained using large values of $\lambda$ to increase the weight of the torques in front of the pure forces (see Section II). The following are some examples, where the algorithm was run with $\lambda=100$ until all the points of the object boundary were selected in order to show the quick convergence. In both cases, it is given the average quality with a $95 \%$ confidence interval for ten trials, and the particular information of the best trial. The average time for the selection of a point and the evaluation of the new grasp was $17 \mathrm{~ms}$ on a Dual Core PC @1.83GHz.

Object 1: Cube modeled with a triangular mesh composed of 21,312 triangles.

- For ten trials (Figure 6)

Final quality $Q: 0.282 \pm 0.020$

Quality after visiting $10 \%$ of the points: $0.252 \pm 0.021$

- For the best trial (Figure 7)

Final quality $Q: 0.322$

Number of visited points: 21,312

Number of point changes improving or maintaining the quality: 251

Number of visited points to obtain $90 \%$ of $Q: 8,041$

Quality after visiting $10 \%$ of the points: 0.212

Object 2: Sausage modeled with a triangular mesh composed of 18,880 triangles.

- For ten trials (Figure 8)

Final quality $Q: 0.222 \pm 0.012$

Quality after visiting $10 \%$ of the points: $0.195 \pm 0.018$

- For the best trial (Figure 9)

Final quality $Q: 0.251$ 

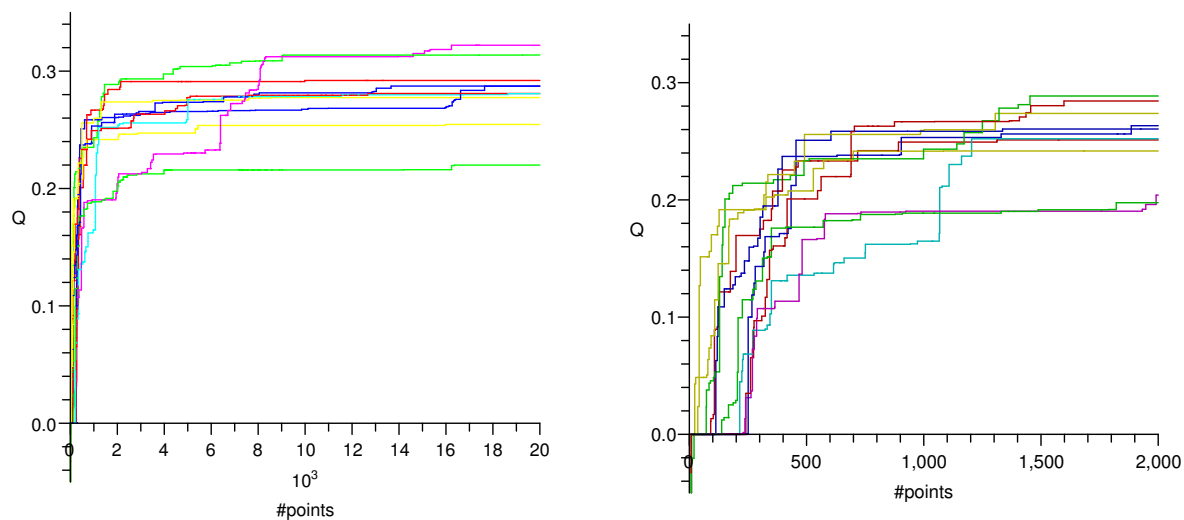

Fig. 6. Cube. Q for ten trials: evolution after visiting all the boundary triangles (left); zoom of the first $10 \%$ of visited triangles (right).
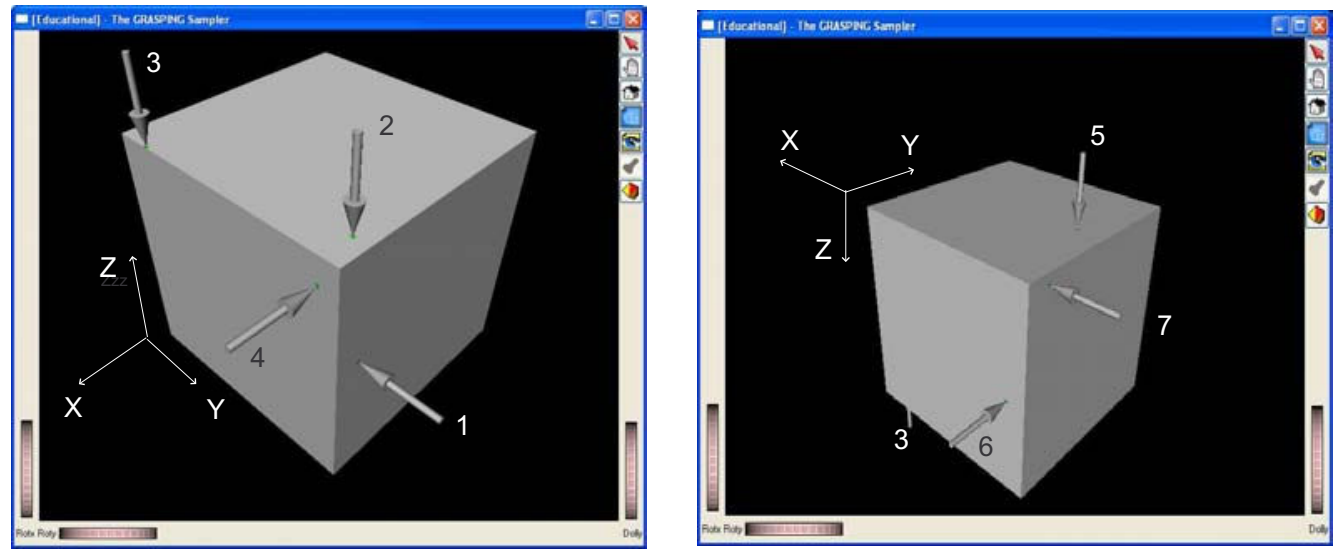

Fig. 7. Cube: Two views of the resulting fixturing (best trial).

Number of visited points: 18,880

Number of point changes improving or maintaining the quality: 69

Number of visited points to obtain $90 \%$ of $Q: 1,923$

Quality after visiting $10 \%$ of the points: 0.219

\section{Discussion AND Conclusions}

The fixturing of objects is a requirement in many practical cases, like for instance during parts manufacturing or during an assembly operation. In this paper a simple yet efficient method has been proposed to find the seven fixturing points needed to produce a form-closure constraint of any freeform object described by a triangular mesh. The method is based on the deterministic sampling of the triangles of the mesh that allows an incremental and uniform covering of the object's surface. Starting with the first seven samples, the procedure iteratively changes one of them by the next sample in the sequence provided it results in a better quality measured in the wrench space. A polyhedral and a free-form object represented by triangular meshes with thousands of triangles were used to illustrate the method performance.

Due to the random nature of the algorithm, it is not possible to predict the computational cost of a grasp search, particularly because the algorithm can be stopped at any time if the current grasp quality is acceptable. Of course, the higher the current quality the more difficult will be to improve it, that is the reason why we include as relevant information in the experiments the number of visited points to obtain the $90 \%$ of the final quality (i.e. the quality after visiting once all the points in the object boundary, which is not initially known), and the quality obtained when $10 \%$ of the object boundary points were visited. The experiments have shown that the approach is more efficient when the object has a smooth shape (i.e. the boundary normal does not change a lot); while for objects with very irregular shapes the approach performance is closer to a pure random selection of points.

The extension of the method to generate force-closure constraints with different number of frictional contacts is currently under development.

\section{REFERENCES}

[1] A. Bicchi, "On the closure properties of robotic grasping," Int. J. Robotics Research, vol. 14, no. 4, pp. 319-344, 1995.

[2] A. Bicchi and V. Kumar, "Robotic grasping and contact: A review," in Proc. IEEE ICRA 2000, 2000, pp. 348-352.

[3] Y. Liu, "Computing n-finger form-closure grasps on polygonal objects," Int. J. Robotics Research, vol. 19, no. 2, pp. 149-158, 2000. 

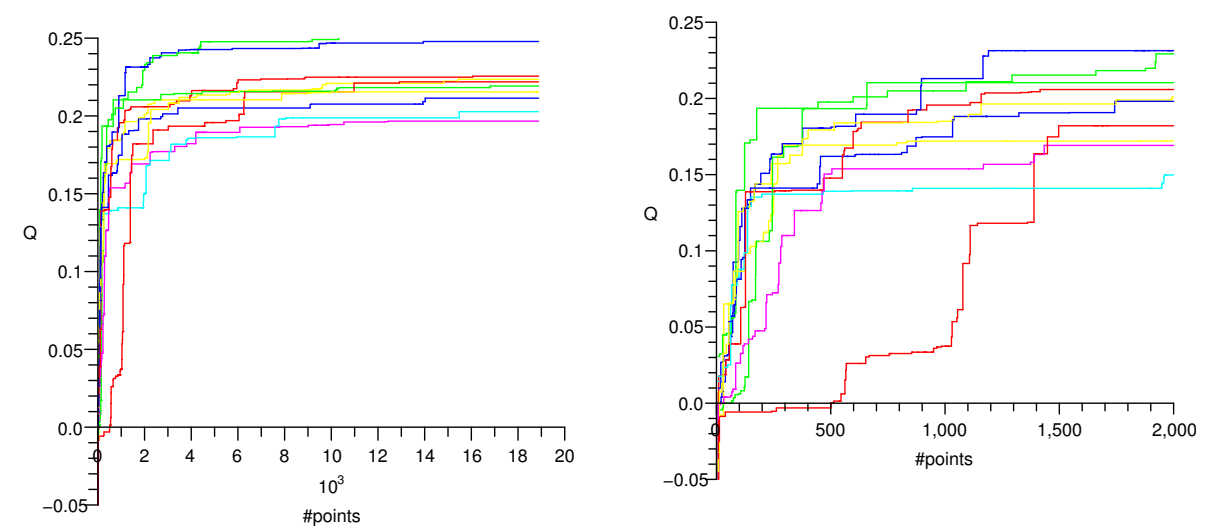

Fig. 8. Sausage. $Q$ for ten trials: evolution after visiting all the boundary triangles (left); zoom of the first 10\% of visited triangles (right).
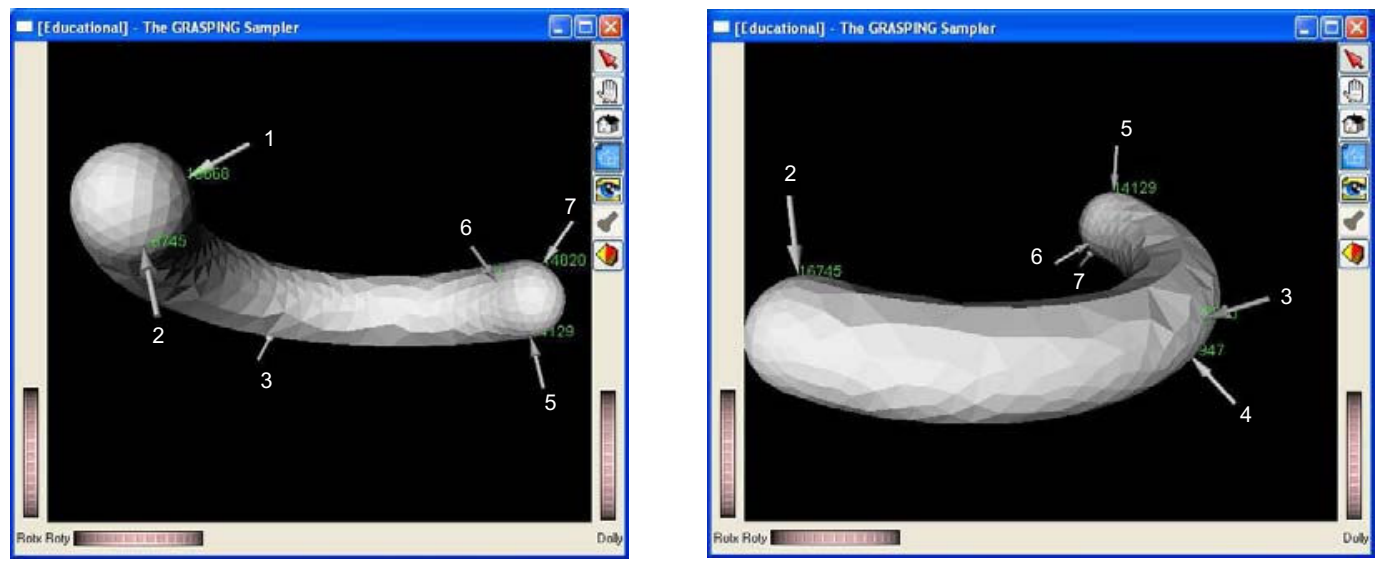

Fig. 9. Sausage: Two views of the resulting fixturing (best trial).

[4] J. Cornellà and R. Suárez, "On computing form-closure grasps/fixtures for non-polygonal objects," in Proc. IEEE Int. Symp. Assembly and Task Planning, ISATP 2005, 2005, pp. 138-143.

[5] N. Niparnan and A. Sudsang, "Computing all force-closure grasps of 2D objects from contact point set," in Proc. IEEE/RSJ IROS 2006, 2006, pp. 1599-1604.

[6] J. Ponce, S. Sullivan, A. Sudsang, J. Boissonat, and J. Merlet, "On computing four-finger equilibrium and force-closure grasps of polyhedral objects," Int. J. Robotics Research, vol. 16, no. 1, pp. 1135, 1997.

[7] D. Ding, Y. Liu, and S. Wang, "Computation of 3-D form-closure grasps," IEEE Trans. Robotics and Automation, vol. 17, no. 4, pp. 515-522, 2001.

[8] X. Zhu and J. Wang, "Synthesis of force-closure grasps on 3-D objects based on the Q distance," IEEE Trans. Robotics and Automation, vol. 19, no. 4, pp. 669-679, 2003.

[9] X. Zhu and H. Ding, "Planning force-closure grasps on 3-D objects," in Proc. IEEE ICRA 2004, 2004, pp. 1258-1263.

[10] Y. Liu, M. Lam, and D. Ding, "A complete and efficient algorithm for searching 3-D form closure grasps in the discrete domain," IEEE
Trans. Robotics, vol. 20, no. 5, pp. 805-816, 2004.

[11] N. Niparnan and A. Sudsang, "Fast computation of 4-fingered forceclosure grasps from surface points," in Proc. IEEE IROS 2004, 2004, pp. 3692-3697.

[12] D. Kirkpatrick, B. Mishra, and C. Yap, "Quantitative Steinitz's theorem with applications to multifingered grasping," Discrete and Computational Geometry, vol. 7, no. 3, pp. 295-318, 1992.

[13] C. Ferrari and J. Canny, "Planning optimal grasps," in Proc. IEEE ICRA 1992, 1992, pp. 2290-2295.

[14] J. Cornellà and R. Suárez, "On 2D 4-finger frictionless optimal grasps," in Proc. IEEE/RSJ IROS 2003, 2003, pp. 3680-3685.

[15] C. Borst, M. Fischer, and G. Hirzinger, "Grasping the dice by dicing the grasp," in Proc. IEEE/RSJ IROS 2003, 2003, pp. 3692-3697.

[16] A. Miller and P. Allen, "GraspIt! a versatile simulator for robotic grasping," IEEE Robotics and Automation Magazine, vol. 11, no. 4, pp. 110-122, 2004.

[17] B. Mishra, "Grasp metrics: Optimality and complexity," in Algorithmic Foundations of Robotics, K. Goldberg, D. Halperin, J. Latombe, and R. Wilson, Eds. A.K. Peters, 1995, pp. 137-166. 